1 Universidade de Ciências Aplicadas de Fulda (Hochschule Fulda) - Fulda, Alemanha. drjensholst@web.de

2 Fundação Oswaldo Cruz (Fiocruz), Escola Naciona de Saúde Pública Sergio Arouca (Ensp) - Rio de Janeiro (RJ), Brasil. giovanel@ensp.fiocruz.br

3 Fundação Oswaldo Cruz (Fiocruz), Escola Naciona de Saúde Pública Sergio Arouca (Ensp) - Rio de Janeiro (RJ), Brasil. andrade.gabriella@gmail. com

\section{Porque não instituir copagamento no Sistema Único de Saúde: efeitos nocivos para o acesso a serviços e a saúde dos cidadãos}

\author{
Why it is a bad idea to implement copayments in the Unified Health \\ System: harmful effects on access to care and people's health
}

Jens Holst1, Ligia Giovanella², Gabriella Carrilho Lins de Andrade ${ }^{\mathbf{3}}$

RESUMO Em tempos de recessão econômica, copagamento é medida aventada para controlar a demanda e reduzir gastos em saúde. O artigo sintetiza pesquisas sobre os efeitos do copagamento. Os resultados evidenciam efeitos deletérios importantes: redução do acesso a medidas de promoção e prevenção, piora na adesão ao tratamento, renúncia ou postergação do uso de serviços, em especial por idosos, doentes crônicos e pessoas de baixa renda, gastos administrativos adicionais, e aumento das desigualdades sociais. Os supostos resultados de eficiência não são comprovados, pelo contrário, os pacientes abdicam de serviços necessários e renunciam à atenção em tempo oportuno, elevando custos assistenciais.

PALAVRAS-CHAVE Financiamento da assistência à saúde. Acesso aos serviços de saúde. Reforma dos serviços de saúde.

ABSTRACT In times of economic recession, copayments are one measure put forward to control demand and reducing public expenditures. The article aims to review the research on the effects of copayments. The results show prominent adverse effects: reduced access to health promotion and prevention services, impaired medication adherence, waiving or postponing health services utilization, particularly reducing access for elderly and chronically ill, additional administrative expenditures and increasing social inequalities in financing and access. Evidence of the assumed efficiency gains is lacking; increasing healthcare costs rather tend to prevent patients from taking out needed services at all or in time.

KEYWORDS Healthcare financing. Health services accessibility. Health care reform. 


\section{Introdução}

Em tempos de recessão e supremacia política conservadora em que as despesas governamentais são consideradas o único vilão e o corte de gastos em políticas sociais, a solução para todos os males, o acesso e a qualidade da atenção à saúde são seriamente ameaçados. A redução de gastos públicos impõe maior participação direta das famílias no custeio de sua atenção, ao mesmo tempo em que a ideia de introduzir copagamento como complemento ao financiamento do Sistema Único de Saúde (SUS), com cobrança de serviços do paciente, volta a rondar os debates nos bastidores dos círculos políticos conservadores.

Com a implementação de copagamentos no SUS, o Brasil correria o risco de cair na esparrela de um dos mitos mais difundidos nas reformas conservadoras de políticas de saúde e repetir os mesmos erros de outros países, tanto industrializados como em vias de desenvolvimento, com consequências nefastas para a equidade no acesso e para o estado de saúde dos brasileiros.

Este artigo tem por objetivo sumariar resultados de pesquisas internacionais que analisaram efeitos da introdução ou da ampliação de copagamentos nos sistemas de serviços de saúde. Inicia com a descrição das modalidades de copagamento e os objetivos declarados para sua introdução. Em seguida, sintetiza as principais consequências do copagamento para o acesso aos serviços de saúde e efeitos sobre a saúde da população. Dentre esses, destaca: a redução do acesso a medidas de promoção e prevenção, a piora na adesão aos tratamentos medicamentosos, a renúncia ou postergação do uso de serviços de saúde, a maior redução do acesso para pessoas idosas e portadores de doenças crônicas, com prejuízos para a saúde e elevação de gastos com esses pacientes, o aumento das desigualdades sociais no acesso e os gastos administrativos adicionais.

\section{Copagamento: definições e objetivos}

Mecanismos de copagamento, em termos gerais, podem ser definidos como a obrigatoriedade do paciente/segurado/usuário arcar com parte dos custos dos serviços de saúde no ato da utilização. Tal mecanismo de obrigatoriedade de participação direta nos custos apresenta diferentes denominações, além de copagamento, como taxa moderadora, participação no custeio (cost-sharing), coparticipação ou, ainda, contrapartida do usuário.

A ideia de fazer o usuário participar diretamente no financiamento dos serviços de saúde é antiga. Adquiriu diferentes ênfases e formas ao longo do tempo, principalmente em períodos de recessão econômica ou de predomínio de governos conservadores e neoliberais, e pode estar presente de uma ou outra forma em distintos sistemas de proteção social à saúde. A existência de copagamento é uma característica dos sistemas de saúde com base no mercado e dos seguros privados de saúde, mas pode também ser observada em sistemas de saúde fundados na universalidade de direitos, para acesso a alguns insumos ou serviços, principalmente medicamentos (SILIANSKY DE ANDREAZZI ET AL., 2011). Além disso, nas décadas de 1980 e 1990, o Banco Mundial costumava impor recomendações específicas para implementar copagamentos na atenção à saúde nos países em desenvolvimento com a suposta finalidade de controlar a demanda e aprimorar a eficiência dos sistemas de saúde (UGÁ, 1997). Diga-se de passagem, que tais recomendações hoje foram relegadas a segundo plano devido a seus efeitos nefastos.

O copagamento pode ser aplicado de diferentes formas, como, por exemplo, imposição de taxa fixa para cada serviço médico ou prescrição dispensada; introdução de taxa variável que represente porcentagem do custo total de um serviço; combinações de quantias fixas e taxas percentuais; e sistema 
de 'franquia anual', ou seja, a fixação de nível mínimo anual para as despesas de medicamentos ou de serviços por paciente, abaixo do qual nenhum reembolso é concedido, sistema utilizado principalmente em seguros privados (RIETVELD; HAAIJER-HUSKAMP, 2003). Alguns autores classificam também como copagamento a exclusão de certos serviços do pacote coberto pelo sistema público de saúde ou pelo seguro social (BUSSE; SCHREYÖGG; HENKE, 2005). No sentido lato, de participação privada nos gastos em saúde, existem copagamentos em quase todos os sistemas de saúde, incluídos os países com sistemas nacionais ou seguros sociais universais de saúde, embora o nível da participação no custeio varie muito de um país a outro e seja menor naqueles com sistemas mais universalisados (GOTTRET; SCHIEBER, 2006).

A tendência de implementar ou aumentar a participação dos pacientes no custeio direto em sistemas públicos de saúde é, em geral, expressão de políticas de cunho neoliberal que invocam a responsabilidade própria do paciente como alternativa ou delimitação frente à suposta tutela do Estado. Nesse sentido amplo, no caso do Brasil, não se pode dizer que o copagamento na saúde seja uma prática completamente desconhecida, dada a importante participação privada nos gastos totais em saúde. A participação dos gastos públicos no total de gastos em saúde no Brasil é muito baixa. Enquanto em países como Alemanha, Canadá, Espanha, França, Itália, Reino Unido, Suécia, mais de $70 \%$ dos gastos totais em saúde são públi$\cos -77,0 \%, 70,9 \%, 70,9 \%, 78,2 \%, 75,6 \%$, $83,1 \%$ e $84,0 \%$, respectivamente (WHO, 2016) -, no Brasil, mais de $50 \%$ dos gastos em saúde são privados, indicando importante participação dos indivíduos e famílias no custeio. Dentre os gastos privados, cerca de metade refere-se a pagamentos diretos no ato da utilização (out of pocket), especialmente para medicamentos. $\mathrm{O}$ modelo de subsídio de medicamentos em farmácias privadas no Programa Farmácia Popular também pode ser considerado copagamento. Entretanto, não há copagamento formalizado no SUS para atenção ambulatorial e hospitalar.

\section{Objetivos do copagamento}

Os partidários do custeio direto dos pacientes no ato da utilização destacam, principalmente, dois objetivos do copagamento: por um lado, a recuperação de custos da atenção médica e o alívio financeiro do sistema ocasionado pelo aporte monetário adicional e, por outro lado, a condução da demanda pretensamente exagerada por serviços médicos, visando a limitar o uso desnecessário, supérfluo ou fútil, e, com isso, controlar o 'risco moral' dos usuários do sistema de saúde. Em nível internacional e, sobretudo, nos países industrializados, o segundo argumento desempenha papel prioritário no debate de reformas para a contenção de gastos públicos em saúde. Na economia da saúde, campo em que se cria a maior parte dos argumentos e com maior quantidade de seus defensores, há importantes correntes que desenvolveram teorias e modelos a favor dos copagamentos com base no denominado risco moral (moral hazard). Conforme esse conceito econômico, o acesso à atenção médica gratuita provocaria uma excessiva utilização dos serviços de saúde, o que, ao final, levaria à redução do bem-estar social geral (PAULY, 1968). O mercado de seguros de saúde falharia em transmitir eficientes sinais de preços para os consumidores. $\mathrm{O}$ acesso gratuito levaria a mudanças no comportamento do consumidor, que estaria disposto a consumir serviços de saúde ao máximo (PAULY, 1968; 1983). Segundo essa teoria, a contrapartida financeira do paciente induziria um uso mais racional dos serviços médicos e contribuiria para reduzir os gastos de saúde.

Em países do sul global, predomina o objetivo de gerar recursos adicionais por meio da contrapartida dos usuários de serviços de 
saúde. Cobrar do paciente uma parte do custo da sua atenção médica diminuiria a proporção financiada por pagamento antecipado seja por meio de impostos gerais num sistema público-estatal, de contribuições sociais num sistema de seguro social ou por meio de prêmios no caso dos seguros privados.

Todavia, quanto maior a proporção do gasto total em saúde financiada de forma antecipada e, portanto, independentemente da utilização, tanto maior a eficiência, equidade e o potencial redistributivo do financiamento de saúde (WHO, 2010). Por conseguinte, todo copagamento não só tem um efeito negativo na justiça social como, acima de tudo, prejudica a saúde das pessoas, pois, em geral, induz à postergação da atenção necessária. Em muitos países, a participação financeira do usuário é acompanhada de sistemas de isenção para proteger os pacientes de menor renda, embora sejam difíceis de implementar e só consigam corrigir parcialmente os efeitos indesejados dos copagamentos (BADASU, 2004; WHO, 2005). Elevados custos administrativos inerentes e dificuldades de execução limitam fundamentalmente a eficácia das medidas de isenção (ROBINSON, 2002).

A avaliação científica crítica das evidências empíricas acumuladas durante as últimas décadas não comprova a hipótese de que os pacientes com proteção financeira de saúde utilizam serviços médicos em demasia por serem gratuitos (HOLST, 2010). Ao contrário e como será demonstrado, os copagamentos tendem a subverter o acesso adequado à atenção à saúde, dado que os pacientes abdicam de serviços necessários e renunciam a recorrer à atenção médica em tempo oportuno.

Bastante conhecido, o filme 'Sicko', de Michael Moore, ilustra com maestria os efeitos perversos da cobertura incompleta de seguros de saúde nos Estados Unidos da América (EUA). Recentemente, um de seus colaboradores deu um depoimento contundente ao The Intercept. Afirma que 'literalmente devo minha vida' a Michael Moore pelo seguro de saúde de alta qualidade sem franquia ou copagamento (zero-deductible) que garante aos seus empregados (SCHWARZ, 2016). Relata que quando observou uma alteração estranha em sua pele, procurou assistência médica imediatamente, por contar com cobertura de seguro saúde na qual o acesso não exigia a necessidade de qualquer copagamento. Assim, um melanoma precocemente diagnosticado foi extirpado, salvando sua vida.

\section{Evidências empíricas dos efeitos do copagamento}

As próximas seções resumem parte das evidências empíricas internacionais sobre os efeitos indesejados e nocivos da participação dos pacientes no custeio, enfocando o impacto do copagamento na promoção e prevenção, no uso de medicamentos e na adesão, na equidade, na atenção médica dos idosos e doentes crônicos.

\section{Redução do acesso a medidas de pro- moção e prevenção}

No contexto atual de empenho para fortalecer a prevenção e promoção à saúde, conhecer os efeitos da participação no custeio da utilização destes serviços certamente adquire especial importância. Estudos realizados nas últimas décadas forneceram amplas evidências comprovando que os copagamentos reduzem o uso de serviços preventivos (CRAWFORD; RAMSEY; SPLINTER, 2004; MANNING ET AL., 1987; SOLANKI; SCHAUFLLER, 1999; TRUDE; GROSSMAN, 2004). Isso é confirmado pela observação que o uso de ações preventivas, como rastreamentos para diagnóstico precoce (screenings) e check-ups, aumenta depois que tais serviços passam a ser isentos de copagamentos e incluídos no rol de serviços cobertos (WEINICK; BEAUREGARD, 1997).

Em sistemas com copagamento, a própria incerteza em saber se haverá copagamento 
e as dúvidas sobre os valores dos encargos financeiros correspondentes reduzem o uso de programas de rastreio tal como mamografias e exames de Papanicolau em grupos socioeconômicos de renda mais baixa (SOMKIN ET AL., 2004).

Certamente, é mais fácil e mais rápido conhecer e avaliar o impacto imediato da contrapartida financeira do usuário no uso de medidas de prevenção do que seus efeitos complexos de longo prazo. Além disso, todas as formas de copagamento de saúde restringem a utilização de serviços de prevenção, porque a participação no custeio para outros serviços afeta o uso de medidas preventivas e promocionais. Um estudo sobre a utilização de quatro medidas preventivas - mamografias, exames de Papanicolau, rastreio da pressão arterial e consultas preventivas -, dentre mais de dez mil empregados de empresas no oeste dos EUA, mostrou que a participação no custeio exerceu efeitos negativos significativos sobre o número de consultas preventivas, realização de Papanicolau e mamografias, enquanto os efeitos sobre o rastreio da pressão arterial foram inconsistentes (SOLANKI; SCHAUfFLER; MILleR, 2000). Solanki, Schauffler e Miller (2000) concluem que eliminar qualquer forma de participação financeira dos pacientes nos custos para serviços de prevenção pode ser um meio relativamente fácil e eficaz de aumentar a utilização de cuidados preventivos clínicos recomendados.

Outra investigação analisou a associação entre o nível de copagamentos e a utilização de terapia ambulatorial para alcoolismo após tratamento de intoxicação por álcool entre cidadãos dos Estados Unidos com seguro privado: o número, duração e intensidade da terapia de seguimento utilizada pelos alcoolistas foram inversamente proporcionais ao nível da contrapartida financeira do usuário. Quanto maior o copagamento, menor uso da terapia (STEIN, 2003).

\section{Redução da adesão ao tratamento medicamentoso}

Um dos terrenos típicos da cobrança de copagamentos são as prescrições de medicamentos. Nos sistemas públicos dos países industrializados e recentemente industrializados, os esforços de conduzir ou moderar a demanda por meio de copagamentos se concentram nos medicamentos (GRESS; NIEBUHR; WASEM, 2005). Os produtos farmacêuticos são considerados serviços de saúde de alta elasticidade-preço, cujo uso está fortemente ligado ao nível de copagamentos (CHANDRA; GRUBER; MCKNIGHT, 2007; LEXCHIN; GROOTENDORST, 2002). Revisões sistemáticas demonstram que maiores níveis de participação dos usuários no custeio estão associados a menores taxas de tratamentos com medicamentos, pior adesão ao tratamento e suspensão mais frequente da terapia. Para cada incremento de $10 \%$ na contrapartida financeira do usuário, observa-se uma redução de $2 \%$ a $6 \%$ dos gastos com medicamentos. Ao mesmo tempo, multiplica-se a utilização de outros serviços tais como consultas médicas e hospitalizações (GOLDMAN; JOYCE; ZHENG, 2007).

Recente revisão sistemática mostrou que, para a população coberta por um sistema público de proteção social, mesmo copagamentos baixos aumentam a probabilidade de não adesão ao tratamento medicamentoso em 11\% (SINNOTT ET AL., 2013).

Em outro estudo, o aumento concomitante dos copagamentos e dos preços de medicamentos acima da taxa de inflação nos EUA, entre 1998 e 2000, incrementou a taxa de medicações não tomadas de $9,5 \%$ para $13,1 \%$ entre beneficiários do Medicare, com impactos notórios no tratamento das pessoas de menor renda (WILSON ET AL., 2005). Ainda nos EUA, análise mostrou que duplicar o valor das menores taxas de copagamentos induz a importante redução na ingestão de diversos tipos 
de medicamentos, sobretudo nos casos de anti-inflamatórios não esteroides (45\%), anti-histamínicos (44\%), hipolipemiantes (34\%), bloqueadores dos receptores $\mathrm{H} 2$ e inibidores da bomba de prótons (33\%), anti-hipertensivos (26\%) e antidiabéticos orais (25\%) (GOLDMAN ET AL., 2004).

Diversas pesquisas evidenciaram elevada taxa de abandono da terapia padrão - ácido acetilsalicílico, beta bloqueadores, estatinas -, decorrente dos copagamentos e depois de alta hospitalar, mesmo dentre pacientes para tratamento de problema tão grave como um ataque cardíaco. Por exemplo, $12 \%$ dos pacientes já tinham deixado de tomar todos os medicamentos e $18 \%$, pelo menos um medicamento prescrito durante o primeiro mês depois do tratamento hospitalar (BENNER ET AL., 2002). O achado mais impressionante e consistente é que são particularmente os pacientes pobres, com menor nível de instrução, e idosos que mostram a pior adesão em consequência dos copagamentos (APPLEGATE, 2002; BENNER ET AL., 2002; LEE; GRACE; TAYLOR, 2006; GIBSON ET AL., 2006).

\section{Renúncia ou postergação de consul- tas médicas}

Na Alemanha, depois da introdução de um copagamento de dez Euros trimestrais para a atenção ambulatorial no Seguro Social de Doença, análises mostraram renúncia ou postergação de uso de serviços de saúde, informadas pelos segurados com variação significativa conforme o grupo socioeconômico. No grupo de menor renda, $67,9 \%$ dos usuários informaram ter postergado ou não ter procurado consulta ambulatorial devido ao copagamento, e no grupo de maior renda, $42,6 \%$. Tal renúncia ao uso de serviços necessários ocorreu apesar de o Seguro Social de Doença alemão definir um teto máximo de participação financeira dos segurados em $2 \%$ de sua renda e em $1 \%$ para os doentes crônicos (RÜCKERT; BÖCKEN; MIELCK, 2008).
Redução do acesso para pessoas idosas e portadores de doenças crônicas com consequências para seu estado de saúde e aumento de gastos com outros serviços

São os pacientes de maior idade e de menor renda, sobretudo, que renunciam à tomada de medicamentos prescritos como consequência do copagamento. Nos EUA, quase a quinta parte dos pacientes idosos e até um quarto dos doentes crônicos renunciaram a aviar uma prescrição por causa da participação no custeio: $16 \%$ dos idosos e $22 \%$ dos doentes crônicos deixaram de tomar diariamente uma parte da dose prescrita, enquanto $12,4 \%$ e $18,5 \%$, respectivamente, reduziram a dose diária pela mesma razão (WILSON ET AL., 2007). É particularmente preocupante que a não adesão induzida pelos copagamentos para remédios seja significativamente mais frequente dentre os portadores de condições crônicas, que necessitam de tratamento constante com perdas para sua saúde (SOUMERAI ET AL., 2006; TAIRA ET AL., 2006).

Evidências empíricas abundantes do efeito nocivo de copagamentos na adesão aos tratamentos existem em muitos outros países além dos EUA (JACKEVICIUS; MAMDANI; TU, 2002; SCHULTZ ET AL., 2005; SOUMERAI ET AL., 2006; WHITE ET AL., 2002). Uma investigação retrospectiva realizada na província canadense de Québec sobre os efeitos da introdução de coparticipação financeira dos usuários para medicamentos produziu resultados impressionantes em pessoas idosas e em pessoas de baixa renda. Depois de um leve aumento dos copagamentos, o consumo de remédios essenciais caiu $9,1 \%$ dentre os idosos e $14,4 \%$ dentre os beneficiários da assistência social. O consumo dos medicamentos não essenciais reduziu-se em $15,1 \%$ dentre idosos, e em 22,4\% dentre os beneficiários da assistência SOcial (TAMBLYN ET AL., 2001).

Ao mesmo tempo, a frequência de eventos adversos relacionados à redução do uso de medicamentos - hospitalização, admissão 
em casas de repouso e mortalidade - subiu de $5,8 \%$ para $12,6 \%$ dentre os beneficiários da assistência social e de $14,2 \%$ para $27,6 \%$ dentre os idosos. A taxa de atendimentos de emergência em decorrência do uso insuficiente de medicamentos imprescindíveis aumentou de 8,5 para 19,9 por 10.000 dentre os idosos e de 69,6 para 123,8 por 10.000 dentre os beneficiários da assistência social (TAMBLYN ET AL., 2001).

É evidente que os gastos relacionados aos tratamentos adicionais decorrentes do não uso facilmente excedem a economia produzida pela participação no custeio daquelas pessoas.

O copagamento afeta particularmente o uso de medicamentos anti-hipertensivos, cardiovasculares e psicotrópicos que os pacientes devem tomar regular e continuamente durante um período longo (REEDER; NELSON, 1985). Isso é especialmente grave no contexto atual de crescente relevância epidemiológica e financeira das doenças crônicas seja em países industrializados ou em países de renda média, emergentes, como o Brasil. Assegurar adequada terapia farmacológica no longo prazo é fundamental para o controle da evolução da doença, medida de prevenção secundária que evita complicações e tem impactos na eficiência, pois previne maiores gastos individuais com serviços de saúde (GAYNOR; LI; VOGT, 2006).

A adesão ao tratamento com cumprimento das prescrições médicas está fortemente associada a resultados mais positivos no estado de saúde com redução de hospitalização e mortalidade (ATELLA ET AL., 2006). Os resultados em saúde melhoram se os pacientes aderem mais ao tratamento. Como mudanças na estrutura e no montante de copagamento têm efeitos na redução da adesão, consequentemente, afetam de maneira negativa o estado de saúde da população. Atella et al. (2006) comprovam evidências de causalidade entre aumento de copagamento e redução da adesão e entre melhor adesão e melhores resultados em saúde.
O impacto que até copagamentos insignificantes podem ter ficou muito evidente durante duas reformas na Itália, ocorridas num lapso de pouco mais de um ano. A primeira aboliu o copagamento e a segunda o reintroduziu. Aquele 'experimento natural' comprovou que a abolição temporária do copagamento para medicamentos induziu a melhora na adesão ao tratamento, efeito que foi revertido depois da reintrodução de pequena contrapartida financeira dos usuários. Com a abolição do copagamento, notavelmente, a taxa de hospitalização dos pacientes hipertensos diminuiu 11,4\% (de $7,9$ para $7,0 \%)$ e a taxa de mortalidade decresceu $6 \%$ (de 3,4\% para 3,2\%) em somente 14 meses, voltando ao nível de partida após a reintrodução do copagamento (ATELLA ET AL., 2006). Os autores sugerem a abolição de copagamentos e argumentam que os gastos adicionais decorrentes da eliminação do copagamento poderiam ser ao menos parcialmente compensados pela redução de custo associada a menores taxas de hospitalização e de mortalidade.

Estudo mais recente realizado na Finlândia também comprovou que menor participação no custeio na fase inicial do tratamento com hipolipemiantes da classe das estatinas está associada com impacto positivo na adesão (HELIN-SALMIVAARA ET AL., 2011). Outro estudo recente na Suécia comprovou os efeitos da participação financeira do usuário na redução da adesão ao tratamento de pacientes com epilepsia (LESÉN ET AL., 2014).

Assim como os pacientes com doença coronária e hipertensão, os portadores de diabetes também tendem a reduzir a dose ou até interromper sua medicação quando têm de pagar do próprio bolso uma parte do custo dos remédios, pondo em risco os avanços reais na melhoria da atenção aos portadores de diabetes mellitus conseguidos nas últimas décadas (SAADDINE ET AL., 2006). Em acordo com as expectativas de redução da demanda, o copagamento restringe a utilização de tiras de glicose e outros insumos e medicamentos 
(ROBLIN ET AL., 2005). O tratamento de diabetes incompleto, insuficiente ou sua interrupção aumenta significativamente o risco de hospitalização e de morte (HO ET AL., 2006).

Ademais, o tratamento insuficiente dos pacientes de doença cardíaca coronariana e de outros problemas vasculares com medicamentos essenciais, como betabloqueadores e hipolipemiantes, produz uma série de resultados indesejados responsáveis por altos custos evitáveis que facilmente superam os gastos relativamente modestos do tratamento farmacológico (SOUMERAI ET AL., 1997, 2006).

Em outras palavras, a economia que se alcança por meio da participação dos pacientes no custeio é muito menor do que os gastos adicionais evitáveis que se incorre para corrigir as consequências indesejadas do copagamento. Estudo com pacientes beneficiários do Medicaid nos Estados Unidos demonstrou, por exemplo, que a restrição da quantidade de prescrições médicas de psicotrópicos reembolsáveis a cada mês para pacientes de menor renda levou à redução entre $15 \%$ e $49 \%$ do uso de medicamentos antipsicóticos, antidepressivos e ansiolíticos - e, consequentemente ao aumento significativo da utilização de serviços psiquiátricos de emergência e clínicas diurnas, o que elevou não só o sofrimento das pessoas afetadas como também os gastos totais desse grupo de pacientes. A despesa per capita para esses pacientes psiquiátricos ambulatoriais ultrapassou a receita conseguida por meio dos copagamentos em mais de 17 vezes, excluída a contabilização de outros custos indiretos (SOUMERAI ET AL., 1994).

\section{Aumento das desigualdades sociais no acesso}

A participação do usuário no custeio no ato da utilização implica intrinsecamente prejuízo para os doentes porque somente afeta as pessoas que precisam de atenção médica e poupa os saudáveis de uma parte do pagamento. Reduzir parte do orçamento total de saúde financiado de forma antecipada, solidariamente, com impostos e contribuições sociais, por meio da introdução de copagamentos afeta a universalidade, pois diminui a profundidade da cobertura, ou seja, o nível em que a atenção é coberta com recursos públicos, com recursos compartilhados.

O copagamento representa uma forma de financiamento regressiva. Ao estabelecer participação em quantidades fixas ou proporcionais ao preço dos serviços produz maior impacto nas pessoas com menor renda, reduzindo, desse modo, os efeitos de redistribuição e aumentando a injustiça social. Revisões sistemáticas mostram que o copagamento em saúde gera importantes efeitos de barreira ao acesso a serviços, particularmente nos grupos de baixa renda e de limitado poder de compra, os quais, em geral, já se destacam por uso menor e insuficiente de serviços de saúde (BECK, 1974; HOLST, 2009; WHITEHEAD ET AL., 2001).

Os efeitos adversos dos pagamentos diretos na equidade são amplamente reconhecidos. Estudos realizados pela Organização para a Cooperação e o Desenvolvimento Econômico (OCDE) em diversos países demonstraram, independentemente das diferentes estruturas institucionais, que o desembolso direto por parte do paciente é a forma de financiamento mais regressiva que existe. Isto é, os mais pobres pagam relativamente mais em comparação à sua renda (RUGER; KIM, 2007). Os próprios defensores da introdução do copagamento reconhecem que a medida afeta diferenciadamente os grupos de renda, prejudicando aqueles menos favorecidos, tanto que, em geral, são introduzidos escalonamentos que dispensam os grupos de menor renda dos pagamentos.

Argumenta-se que copagamentos escalonados conforme classes de renda incidiriam positivamente na equidade. Teoricamente seria possível introduzir um sistema de copagamentos escalonados conforme a renda das famílias para aliviar a carga financeira 
dos grupos socioeconômicos menos favorecidos. Assim, também teoricamente, o escalonamento poderia mitigar a regressividade do financiamento de saúde, embora certamente não permita dirimir as desigualdades, porque sempre afeta os mais doentes, corroendo a solidariedade entre saudáveis e enfermos (RAMSAY, 1998).

Em vez de reduzir a utilização supostamente exagerada de serviços médicos e evitar uma pretensa perda de bem-estar social, os copagamentos minam a confiança dos cidadãos na redistribuição e solidariedade. Apesar de toda a retórica, a contrapartida do usuário favorece aos saudáveis e às pessoas de maior renda à custa dos doentes e dos mais pobres.

\section{Gastos administrativos adicionais}

Na prática, a introdução de copagamentos e de um sistema de escalonamento implica procedimentos administrativos complexos de difícil aplicação (WITTER, 2002). Isso é particularmente válido para os sistemas estatais financiados por meio de impostos, como o SUS, que, em geral, carecem de informação confiável sobre a situação de renda dos usuários - a menos que exista um sistema fiscal sofisticado e transparente, o que é pouco frequente.

A participação no custeio dos pacientes é cobrada no momento e local da utilização dos serviços médicos, o que significa que os prestadores têm de dispor de informações realistas sobre a renda dos usuários para poder cobrar o copagamento correspondente. Além disso, um sistema de copagamentos escalonados conforme a renda aumenta ainda mais os custos administrativos incorridos pela cobrança. Com escalonamento, esses custos podem consumir de $40 \%$ a $60 \%$ da receita gerada pelo copagamento (WITTER, 2002) ou até exceder os benefícios financeiros (FIEDLER; SUAZO, 2002).

Em suma, um mecanismo de isenção que seria indispensável para proteger os pobres das consequências indesejadas do copagamento aumentaria ainda mais os requisitos burocráticos e a necessidade de controle social sobre a sua aplicação.

Ainda que mesmo pequenas elevações no copagamento reduzam o uso e adesão aos tratamentos, o alívio do financiamento do sistema de saúde por meio da recuperação de custos da atenção médica por gerar novas receitas - o outro objetivo da introdução do copagamento - somente é alcançado com copagamentos altos e substantivos em virtude dos gastos administrativos decorrentes; ademais dos gastos adicionais com uso de outros serviços por postergação da procura.

Para o SUS, a introdução de um sistema escalonado de participação financeira dos pacientes significaria uma enorme carga administrativa adicional desde o nível central até o local, incluindo hospitais e centros de saúde públicos, porque teriam de implementar procedimentos de avaliação da capacidade de pagamento dos pacientes, criar mecanismos de cobrança e controle e organizar os correspondentes fluxos financeiros. Para o SUS, a implementação de um sistema de copagamentos escalonados não somente demandaria desafio administrativo maior como também poderia produzir pontos de corrupção e ampliar o risco de imposição de pagamentos adicionais 'por debaixo da mesa'.

\section{Considerações finais}

Com poucas exceções, a participação no custeio nos países europeus com sistemas universais aplica-se, em geral, somente aos serviços de porta de entrada e menos custosos, como consultas médicas ambulatoriais e medicamentos. Para tratamentos mais complexos, e mais dispendiosos, em geral, não há contrapartida financeira do usuário, ou ela é mínima em relação ao custo total. Nesses países, a imposição aos doentes crônicos e graves de um sistema efetivo de 
copagamentos altos é socialmente rejeitada e tem custo político muito elevado.

A instituição do copagamento como medida moderadora da utilização dos serviços de saúde parte do pressuposto que a demanda em saúde é excessiva - ações desnecessárias seriam consumidas por iniciativa do paciente - e é elástica, ou seja, reduz-se com a elevação dos preços. Todavia, a elasticidade-preço da demanda por ações de saúde varia segundo a faixa de renda do usuário. Além disso, a demanda em saúde é predominantemente secundária, ou seja, induzida pelos prestadores e definida pelo médico, não pelo paciente. $\mathrm{O}$ nível da elasticidade da demanda de serviços de saúde frente aos preços, guarda correlação com o nível de renda das pessoas (REMLER; ATHERLY, 2003) e, sobretudo, situações de doenças graves demonstram elevado grau de inelasticidade (MANNING ET AL., 1987).

Por se tratar de questão de sobrevivência, a demanda não diminui com o aumento de preços: o demandante aloca o máximo de recursos financeiros que pode disponibilizar. No entanto, quando há insuficiência da renda, a demanda torna-se elástica. E quanto mais baixa a renda, maior a elasticidade da demanda por serviços de saúde, rapidamente atingindo o patamar da impossibilidade absoluta, o que significa dizer que pacientes necessitados ficarão sem a assistência devida (CHANDRA ET AL., 2007).

A introdução do copagamento, em lugar de racionalizar o comportamento dos usuários, é mais provável que contribua para conter a demanda necessária, resultando em subatenção para determinados grupos, do que para conter a demanda supérflua, referente à sobreutilização (OECD, 1995).

A participação no custeio é geralmente considerada uma medida eficiente para baixar os gastos em saúde e reduzir o pretenso 'risco moral' dos pacientes. No entanto, como sumarizado neste artigo, cada vez mais evidências empíricas relegam a estratégia de implementar copagamentos ao império dos mitos e das meias verdades nas políticas de saúde. Apesar da contrapartida do usuário, à primeira vista, mobilizar recursos adicionais para prestação dos serviços, análises mais precisas provam o contrário: existe uma série de efeitos indesejados e nocivos da participação no custeio em saúde sobre o acesso e o estado de saúde da população como consequência da postergação ou renúncia ao uso de serviços com custos adicionais.

Uma ação de saúde não realizada, i.e., 'poupada', não se constitui automaticamente em benefício para o financiamento do sistema de saúde. A postergação da utilização de serviços de saúde tende a piorar o estado de saúde dos pacientes e, assim, provocar gastos mais elevados. Este efeito bumerangue é particularmente frequente dentre os doentes crônicos e, sobretudo, nos grupos sociais de menor renda. Sistemas de isenções tendem a falhar e não compensam os efeitos indesejados dos copagamentos, exigem esforços burocráticos adicionais e ocasionam pontos de corrupção.

A cobrança de copagamentos causa gastos administrativos adicionais nos serviços de saúde para instituir guichês de cobrança e medidas de controle da arrecadação de recursos, sobretudo quando se implementa um sistema de isenções que requer a estratificação da população por grupos de renda. Além disso, a cobrança da participação do usuário traz consigo o perigo de estabelecer e capilarizar pontos de corrupção que aumentam ainda mais as desigualdades existentes.

A participação financeira direta no ato da utilização não consegue produzir maior equidade. A equidade no financiamento em saúde resulta da composição das fontes de financiamento. Nos serviços nacionais de saúde de acesso universal com financiamento fiscal, a equidade se alcança por meio de um sistema de tributação progressiva em que os impostos incidentes sobre a renda têm maior peso. Nos seguros sociais de saúde, a equidade resulta da contribuição social proporcional à renda e da paridade na contribuição de 
empregadores e trabalhadores. No Brasil, o sistema de tributação é altamente regressivo, com predomínio de receitas provenientes de impostos sobre o consumo, tais como os impostos sobre valor agregado ou sobre circulação de mercadorias, que incidem mais do que proporcionalmente sobre os orçamentos das famílias mais pobres (OECD; ECLAC, 2011).

As tentativas de distinguir entre demandas necessárias e desnecessárias são irreais e estão fadadas ao fracasso, pois não refletem adequadamente a realidade das pessoas que necessitam de atenção médica e serviços de saúde. Assim, a teoria econômica de risco moral dos usuários prescinde de justificativa ou fundamentos empíricos. A participação do usuário no financiamento implica alto risco para o acesso universal à saúde, a justiça social e, principalmente, para a saúde das pessoas.

\section{Colaboradores}

Jens Holst realizou a revisão bibliográfica, concebeu e realizou as análises e interpretação e redigiu a primeira versão do manuscrito; Ligia Giovanella contribuiu para a concepção, planejamento, elaboração do manuscrito e revisão crítica do conteúdo; Gabriella Carrilho Lins de Andrade contribuiu para a revisão e participou da aprovação da versão final do manuscrito.

\section{Referências}

APPLEGATE, W. B. Elderly patients' adherence to statin therapy. JAMA, Chicago, v. 288, n. 4, p. 495-497, 2002.

ATELLA, V. et al. Drug compliance, co-payment and health outcomes: evidence from a panel of Italian patients. Health Economics, Nova York, v. 15, n. 9, p. 875-892, 2006.

BECK, R. G. The effects of co-payment on the poor. The Journal of Human Resources, Madison, v. 9, n. 1, p. 129-142, 1974.

BENNER, J. S. et al. Long-term persistence in use of statin therapy in elderly patients. JAMA, Chicago, v. 288, n. 4, p. 455-461, 2002.

BUSSE, R.; SCHREYÖGG, J.; HENKE, K. Regulation of pharmaceutical markets in Germany: improving efficiency and controlling expenditures? The International Journal of Health Planning and Management, Chichester, v. 20, n. 4, p. 329-349, 2005.

CHANDRA, A.; GRUBER, J.; MCKNIGHT, R. Patient cost-sharing, hospitalization offsets, and the design of optimal health insurance for the elderly. NBER Working Paper 12972. Cambridge: National Bureau of Economic Research, 2007. Disponível em: < http://www.nber.org/ papers/w12972.pdf> Acesso em: 18 out. 2016.

CRAWFORD, S. A.; RAMSEY, C. N.; SPLINTER, G. L. It's health care, not welfare. Final Report. Oklahoma City: University of Oklahoma Health Sciences Center, 2004. Disponível em: <http://www.statecoverage.org/ files/Its\%20Health\%20Care\%20Not\%20Welfare $\% 20$ -\%20Final\%20Report.pdf> Acesso em: 15 out. 2016. 
FIEDLER, J. L.; SUAZO, J. Ministry of Health user fees, equity and decentralization: lessons from Honduras. Health Policy and Planning, Oxford, v. 17, n. 4, p. 362377, 2002.

GAYNOR, M.; LI, J.; VOGT, W. Is drug coverage a free lunch? Cross-price elasticities and the design of prescription drug benefits. NBER Working Paper 12758. Cambridge: National Bureau of Economic Research, 2006. Disponível em: <http://www.nber.org/ papers/w12758.pdf $>$. Acesso em: 10 out. 2016.

GIBSON, T. B. et al. Impact of statin copayments on adherence and medical care utilization and expenditures. The American Journal of Managed Care, Old Bridge, v. 12, n. 12, p. 11-19, 2006.

GOLDMAN, D. P. et al. Pharmacy benefits and the use of drugs by the chronically Ill. JAMA, Chicago, v. 291, n. 19, p. 2344-2350, 2004.

GOLDMAN, D. P.; JOYCE, G. F.; ZHENG, Y.

Prescription drug cost sharing. Associations with medication and medical utilization and spending and health. JAMA, Chicago, v. 298, n. 1, p. 61-69, 2007.

GOTTRET, P.; SCHIEBER, G. Health Financing Revisited. A Practioner's Guide. Washington, DC: The World Bank, 2006.

GREß, S.; NIEBUHR, D.; WASEM, J. Marktzugang und Preisbildung auf Arzneimittelmärkten im internationalen Vergleich. Diskussionsbeiträge aus dem Fachbereich Wirtschaftswissenschaften, Alfried Krupp von Bohlen und Halbach-Stiftungslehrstuhl für Medizinmanagement. Essen: Universität DuisburgEssen, 2005

HELIN-SALMIVAARA, A. et al. Impact of out-ofpocket expenses on discontinuation of statin therapy: a cohort study in Finland. Journal of Clinical Pharmacy and Therapeutics, Oxford, v. 37, n.1, p. 58-64, 2011.

HO, M. P. et al. Effect of medication nonadherence on hospitalization and mortality among patients with diabetes mellitus. JAMA, Chicago, v. 166, n. 17, p. 18361841, 2006.
HOLST, J. Zuzahlungen: Praxisgebühr trifft Arme. Deutsches Arzteblatt, Colônia, v. 106, n. 3, p. A-65/B-59 /C-59, 2009

Patient cost sharing: reforms without evidence. Theoretical considerations and empirical findings from industrialized countries. Discussion Paper SP I 2010303. FG Public Health. Berlin: Wissenschaftszentrum Berlin für Sozialforschung (WZB), 2010. Disponível em: <https://bibliothek.wzb.eu/pdf/2010/i10-303.pdf $>$. Acesso em: 10 out. 2016.

JACKEVICIUS, C. A.; MAMDANI, M.; TU, J. V. Adherence with statin therapy in elderly patients with and without acute coronary syndromes.

JAMA,Chicago, v. 288, n. 4, p. 462-467, 2002.

LEE, J. K.; GRACE, K. A.; TAYLOR, A. J. Effect of a pharmacy care program on medication adherence and persistence, blood pressure, and low-density lipoprotein cholesterol: a randomized controlled trial. JAMA, Chicago, v. 296, n. 21, p. 2563-2571, Dec. 2006.

LESÉN, E. et al. Is the level of patient co-payment for medicines associated with refill adherence in Sweden? European Journal of Public Health, Weinheim,v. 24, n. 1, p. 85-90, Feb. 2014.

LEXCHIN, J.; GROOTENDORST, P. The effects of prescription drug user fees on drug and health services use and health status: a systematic review of evidence. International Journal of Health Services, Thousand Oaks, v. 34, n. 1, p. 101-122, 2004.

MANNING, W. G. et al. Health insurance and the demand for Medical Care: evidence from a randomized experiment. The American Economic Review, Nashville, v. 77, n. 3, p. 251-277, 1987.

NACI, H. Cost-related medication non adherence among elderly and disabled Medicare beneficiaries. A national survey 1 year before the Medicare Drug Benefit. Archives of Internal Medicine, Chicago, v. 166, n. 17, p. 1829-1835, 2006.

ORGANISATION FOR ECONOMIC CO-OPERATION AND DEVELOPMENT (OECD). Policies to improve 
the performance of health care systems. In: New Directions in Health Policy. Health Policy Studies. Paris, 1995. p. 37-67.

ORGANISATION FOR ECONOMIC CO-OPERATION AND DEVELOPMENT (OECD); ECONOMIC COMMISSION FOR LATIN AMERICA AND THE CARIBBEAN (ECLAC). Revenue statistics in Latin America 1990-2010. Paris: OECD; Santiago: ECLAC, 2011. $202 \mathrm{p}$.

PAULY, M. V. The economics of moral hazard: comment. The American Economic Review, Nashville, v. 58, n. 3, p. 531-537, 1968.

More on moral hazard. Journal of Health

Economics, Amsterdam, v. 2, n. 1, p. 81-86, 1983.

RAMSAY, C. Medical savings accounts: universal, accessible, portable, comprehensive health care for Canadians. Vancouver BC: The Fraser Institute. 1998. 40 p.

REEDER, C. E.; NELSON, A. A. The differential impact of copayment on drug use in a Medicaid population. Inquiry, v. 22, n. 4, p. 396-403, Winter 1985.

REMLER, D.; ATHERLY, A. Health status and heterogeneity of cost-sharing responsiveness: how do sick people respond to cost-sharing? Health Economics, Chichester, v. 12, n. 4, p. 269-280, Apr. 2003.

RIETVELD, A.; HAAIJER-RUSKAMP, F. Policy options for cost containment of pharmaceuticals. In: DUKES, G. et al. (Org.). Drugs and money. Prices, affordability and cost containment. Amsterdam: IOS Press ; Genebra: World Health Organization, 2003. p. 29-54.

ROBINSON, J. C. Renewed emphasis on consumer cost sharing in health insurance benefit design. Health Affairs Suppl., Millwood, v. 21, p. w139-w154, 2002.

ROBLIN, D. W. et al. Effect of increased cost-sharing on oral hypoglycemic use in five managed care organizations: how much is too much? Medical Care, Philadelphia, v. 43, n. 10, p. 951-959, 2005.

RÜCKERT, I-M.; BÖCKEN, J.; MIELCK, A. Are German patients burdened by the practice charge for physician visits ('Praxisgebuehr')? A cross sectional analysis of socio-economic and health related factors. BMC Health Services Research, v. 8, n. 232, p. 1-13, Nov. 2008.

RUGER, J. P.; KIM, H. J. Out-of-pocket healthcare spending by the poor and chronically ill in the Republic of Korea. American Journal of Public Health, Washington, DC, v. 97, n. 5, p. 804-811, 2007.

SAADDINE, J. B. et al. Improvements in diabetes processes of care and intermediate outcomes: United States, 1988-2002. Annals of International Medicine, Philadelphia, v. 144, n. 7, p. 465-474, Apr. 2006.

SCHULTZ, J. S. et al. Determinants of compliance with statin therapy and low-density lipoprotein cholesterol goal attainment in a managed care population. The American Journal of Managed Care, Old Bridge, v. 11, n. 5, p. 306-312, 2005.

SCHWARZ, J. "Where to invade next" is the most subversive movie Michael Moore has ever made. The Intercept. 2016. Disponível em: <https://theintercept. com/2016/02/10/where-to-invade-next-is-the-mostsubversive-movie-michael-moore-has-ever-made>. Acesso em: 16 set. 2016.

SILIANSKY DE ANDREAZZI, M. F. et al.

Copagamentos no sistema público brasileiro: anomalia ou tendência? Serviço Social \& Sociedade, São Paulo, v. 105, n. 6, p. 89-109, jan./mar. 2011.

SINNOTT, S. et al. The effect of copayments for prescriptions on adherence to prescription medicines in publicly insured populations; A systematic review and meta-analysis. PLOS One, San Francisco, v. 8, n. 5, p. 1-11, 2013.

SOLANKI, G.; SCHAUFFLER, H. H. Cost-sharing and the utilization of clinical preventive services. American Journal of Preventive Medicine, Nova York, v. 17, n. 2, p. 127-133, 1999.

SOLANKI, G.; SCHAUFFLER, H. H.; MILLER, L. S.

The direct and indirect effects of cost-sharing on the use of preventive services. Health Services Research, 
Chicago, v. 34, n. 6, p. 1331-1350, 2000.

SOMKIN, C. P. et al. The effect of access and satisfaction on regular mammogram and papanicolaou test screening in a multiethnic population. Medical Care, Philadelphia, v. 42, n. 9, p. 914-926, 2004.

SOUMERAI, S. B. et al. Effects of limiting Medicaid drug-reimbursement benefits on the use of pychotropic agents and acute mental health services by patients with schizophrenia. The New England Journal of Medicine, Boston, v. 331, n. 10, p. 650-655, 1994.

SOUMERAI, S. B. et al. Adverse outcomes of underuse of ß-blockers in elderly survivors of acute myocardial infarction. JAMA, Chicago, v. 277, n. 2, p. 115-121, jan. 1997.

STEIN, B. D. Drug and alcohol treatment services among privately insured individuals in managed behavioral health care. 2003. 82 f. Dissertação (Philosophy in Policy Analysis) - RAND Graduate School, Santa Monica, 2003.

TAIRA, D. A. et al. Copayment level and compliance with antihypertensive medication: analysis and policy implications for managed care. The American Journal of Managed Care, Old Bridge, v. 12, n. 11, p. 678-683, 2006.

TAMBLYN, R. et al. Adverse events associated with prescription drug cost-sharing among poor and elderly persons. JAMA, Chicago, v. 285, n. 4, p. 421-429, 2001.

TRUDE, S.; GROSSMAN, J. Patient cost-sharing innovations: promises and pitfalls. Issue Brief Report No. 75, Washington, DC: Center for Studying Health System Change, 2004.

UGÁ, M. A. D. Propostas de reforma do setor saúde nos marcos do ajuste macroeconômico. 1997. 258 p. Tese (Doutorado em Saúde Coletiva) - Universidade do Estado do Rio de Janeiro, Rio de Janeiro, 1997.

WEINICK, R. M.; BEAUREGARD, K. M. Women's use of preventive screening services: a comparison of HMO versus fee-for-service enrollees. Medical Care Research and Review, Thousand Oaks, v. 54, n. 2, p. 176-199, 1997.
WILSON, I. B. et al. Cost-related skipping of medications and other treatments among Medicare beneficiaries between 1998 and 2000. Journal of General Internal Medicine, Philadelphia, v. 20, n. 8, p. 715-720, 2005

WORLD HEALTH ORGANISATION (WHO). Make every mother and child count. Genebra: World Health Report, 2005.

Global Health Observatory data repository. Health expenditure ratios, by country, 1995-2014. Genebra, 2016. Disponível em: <http://apps.who.int/ gho/data/node.main.75?lang=en>. Acesso em: 15 set. 2016.

Health systems financing: the path to universal coverage. World Health Report 2010. Genebra, 2010. Disponível em: <http://apps.who.int/iris/ bitstream/10665/44371/1/9789241564021_eng.pdf>. Acesso em: 7 out. 2016

WHITE, T. J. et al. Patient adherence with HMG reductase inhibitor therapy among users of two types of prescription services. Journal of Managed Care Pharmacy, Alexandria, v. 8, n. 3, p. 186-191, 2002.

WHITEHEAD, M.; DAHLGREN, G.; EVANS, T. Equity and health sector reforms: can low-income countries escape the medical poverty trap? The Lancet, Londres, v. 358, n. 9284 , p. 833-836, 2001.

WILSON, I. B. et al. Physician-patient communication about prescription medication. Nonadherence: a 50-state study of America's seniors. Journal of General Internal Medicine, Philadelphia, v. 22, n. 1, p. 6-12, jan. 2007.

WITTER, S. Health financing in developing and transitional countries. Briefing paper for Oxfam GB. York: The University of York, 2002.

Recebido para publicação em dezembro de 2016

Versão final em dezembro de 2016

Conflito de interesses: inexistente

Suporte financeiro: não houve 